\title{
APLICAÇÃO DA TEORIA DO CAOS EM DADOS DE CONCENTRAÇÃO DE MATERIAL PARTICULADO INALÁVEL NA ATMOSFERA
}

\author{
W. H. PRIETO ${ }^{1}$, E. TOMAZ ${ }^{1}$ e M. A. CREMASCO ${ }^{1}$ \\ ${ }^{1}$ Universidade Estadual de Campinas, Faculdade de Engenharia Química \\ E-mail para contato: wesley@feq.unicamp.br
}

\begin{abstract}
RESUMO - As altas concentrações de material particulado inalável $\left(\mathrm{PM}_{10}\right)$, nos grandes centros urbanos, são responsáveis por desencadearem problemas respiratórios, entre outros problemas. $\mathrm{O}$ controle dessas emissões não é trivial, pois existem diversos fatores que influenciam as concentrações de tais partículas na atmosfera, caracterizando a descrição do fenômeno de poluição como não linear, sensível às condições iniciais e, portanto, caótico. O objetivo deste trabalho é do avaliar a presença de caos em dados de variação temporal de concentração de $\mathrm{PM}_{10}$ nos anos de 1998 a 2000 e 2010 a 2013, obtidos no Parque Ibirapuera, em São Paulo. Os resultados dos invariantes caóticos, entropia de Kolmogorov e dimensão de correlação, advindos de atratores estranhos, apontam a natureza caótica do estudo em análise.
\end{abstract}

\section{INTRODUÇÃO}

Nos últimos anos, tem-se verificado o aumento da preocupação de órgãos ambientais em monitorar e controlar os níveis de poluentes atmosféricos e neste cenário destacam-se os materiais particulados inaláveis $\left(\mathrm{PM}_{10}\right)$ advindos, principalmente, de fontes industriais (plantas químicas, geração de energia) e urbanas (emissões veiculares). Nas grandes metrópoles, altas concentrações de $\mathrm{PM}_{10}$ são os responsáveis pela redução da visibilidade e por desencadearem diversos problemas respiratórios nos seres vivos (Pope et al., 2002). Movidos pela crescente necessidade de controle destas emissões, pesquisadores do mundo inteiro, muitas vezes financiados por órgãos governamentais, esforçam-se na compreensão da dispersão das diversas categorias de materiais particulados e no desenvolvimento de indicadores de qualidade do ar. Diversas abordagens considerando as combinações lineares das interações entre os poluentes atmosféricas foram aplicadas (Hellebust et al., 2010; Guo, 2011) e, desta forma, negligenciaram as complexas interações não lineres presentes no fenômeno.

$\mathrm{Na}$ intenção de estudar a dispersão de materiais particulados atmosféricos, alguns trabalhos aplicam a Análise Fatorial Dinâmica (DFA) como ferramenta para decompor uma série temporal multivariada em combinações lineares dos fatores majoritários e suas variações temporais comuns associadas (Yu et al., 2013). Contudo, analisar as características estocásticas e suas relações associadas não permite avaliar a inteireza de todas as, já solidificadas, relações dinâmicas não lineares presentes nos sistemas atmosféricos (Lorenz, 1963; Tsonis, 1992), sendo assim a análise de caos 
determinístico surge como metodologia alternativa na elucidação destes fenômenos. Em linhas gerais, a análise de caos é aplicada a processos dissipativos cuja evolução temporal é não linear, aperiódica a longo prazo, determinístico e extremamente sensível às condições iniciais (Savi, 2004). Um fenômeno que possua estas características fatalmente apresentará, durante sua evolução temporal, uma contração no volume do espaço de fases e suas linhas de fluxo dependerão das condições iniciais (Ruelle e Takens, 1971) definindo o chamado atrator estranho ou atrator caótico. Dentre os invariantes abstraídos das características topológicas do atrator podem ser citados a entropia de KolmogorovSinai (Ruelle, 1989) e dimensão de correlação (McCauley, 1990).

Nesta tendência, o presente trabalho aplica os invariantes caóticos entropia de Kolmogorov e dimensão de correlação à dados de variação temporal de concentração de material particulado inalável $\left(\mathrm{PM}_{10}\right)$ objetivando identificar características não lineares. Os dados utilizados se referem aos anos de 1998 à 2000 e 2010 à 2013 obtidos na Estação de Monitoramento da CETESB localizada no Parque Ibirapuera na cidade de São Paulo.

\subsection{Atratores Estranhos}

Atrator é o nome dado a região do espaço de fases para o qual evoluem, passado um tempo infinito, as soluções (ou trajetórias) de um determinado sistema (Vander Stappen, 1996) caracterizando um evento dinâmico (Briongos e Soler, 2004). O atrator estranho é aquele que está inserido em um espaço de n-dimensões e sua trajetória nunca retorna a um estado anterior (Van den Bleek e Schouten, 1993) não havendo cruzamento das linhas de fluxo. Takens, em 1981, postulou uma metodologia simplifica para a obtenção do atrator. O método ficou conhecido como método de Takens ou método dos atrasos temporais e consiste na reconstrução de vetores $\lambda_{\mathrm{i}}$, com $n$ dimensões, obtidos de uma série temporal, em que o vetor é representado pela Equação 1.

$$
\lambda_{i}=\left[\varphi\left(t_{i}\right), \varphi\left(t_{i}+\theta\right), \varphi\left(t_{i}+2 \theta\right), \ldots, \varphi\left(t_{i}+(n-1) \theta\right)\right]
$$

Sendo $\lambda_{\mathrm{i}}$ uma série temporal qualquer para $\lambda_{i}=\lambda\left(t_{i}\right), i=1, N, \theta$ é o tempo de atraso e $n$ a dimensão de imersão. Muito embora o atrator obtido não seja idêntico ao real, o Teorema de Takens garante que suas propriedades topológicas são preservadas.

\subsection{Dimensão de Correlação}

Segundo Vander Stapen (1996) a dimensão de correlação $\left(D_{2}\right)$ mede a complexidade global do sistema por meio da quantificação do grau de heterogeneidade do atrator. Para a sua estimativa, pode ser utilizado o algoritmo proposto Grassberger e Procaccia (1983) na forma

$$
D_{2}=-\lim _{N \rightarrow \infty}\left(\lim _{\omega \rightarrow 0} \frac{\log C(\omega)}{\log (\omega)}\right)
$$

na qual $C(\omega)$ é função de correlação com valor médio calculado sobre todos os pontos $x_{j}$ do atrator. Este método é também conhecido como Método da Integral de Correlação, e se consagrou devido sua facilidade de implementação. 


\section{9 a 22 de outubro de 2014 \\ Florianópolis/SC}

\subsection{Entropia de Kolmogorov}

Similar ao conceito termodinâmico clássico, a entropia de Kolmogorov $(K)$ avalia o grau de desorganização ou imprevisibilidade de um sistema, porém sua dinâmica se baseia na quantificação da taxa de perda de informações ao longo do atrator ou na previsibilidade de rota de um determinado ponto arbitrário (Castilho, 2011). Uma das maneiras de se obter o valor de $K$ é por aproximação da determinação da entropia de verossimilhança e do passo de tempo $\left(\tau_{S}\right)$, este obtido pela frequência de amostragem $\left(f_{s}\right)$ como $\tau_{S}=1 / f_{s}$. O valor de $K_{M L}$ (Schouten et al., 1994) advém de

$$
K \approx K_{M L}=\frac{1}{\tau_{S}} \ln \left(1-\frac{1}{b}\right)
$$

\section{MÉTODOS}

As séries temporais utilizadas neste trabalho foram exportadas da plataforma eletrônica da CETESB (Companhia de Tecnologia de Saneamento Ambiental). Os dados se referem à concentração de material particulado inalável $\left(\mathrm{PM}_{10}\right)$ medida nos anos de 1998, 1999, 2000, 2010, 2011, 2012 e 2013 na estação de monitoramento do Ibirapuera na região metropolitana de São Paulo. A frequência de amostragem é de 1 hora, sendo 8760 o número total de pontos de cada série,

Para a reconstrução dos atratores no espaço de fases foi utilizado o método de decomposição em valores singulares (SVD) (Broomhead e King, 1986). No cálculo da entropia de Kolmogorov foi aplicado o método de verossimilhança (Schouten et al., 1994). A dimensão de correlação foi calculada pelo método de Grassberger e Procaccia (1983). Todos os métodos foram implementados por Moura (2013) em um pacote computacional utilizando a linguagem da plataforma Matlab $^{\circledR}$. O software utilizado foi MATLAB R2012b.

\section{RESULTADOS E DISCUSSÕES}

\subsection{Atratores Estranhos e Entropia de Kolmogorov}

Antes de qualquer abordagem que vise a caracterização do caos, é passo primordial a reconstrução do atrator para que se possa abstrair informações ou propriedades de uma determinada série temporal. Da Equação1, nota-se que quando ocorre a variação de $i$ até $N$ para cada instante $t_{i}$, associado a um ponto das coordenadas $\varphi\left(t_{\mathrm{i}}\right), \varphi\left(t_{i}+\theta\right), \ldots, \varphi\left(t_{i}+(n-1) \theta\right)$ no espaço de imersão, é possível obter a trajetória reconstruída no espaço de fases. Os diagramas do espaço de fases presente na Figura 1 foram confeccionados utilizando as propriedades da matriz de covariância para esboçar o grau de independência de cada variável obtendo, assim, variáveis não relacionadas. Este método (decomposição em valores singulares (SVD) (Broomhead e King, 1986)) parte da seleção e separação das frequências características dos ruídos da série original e, desta forma, permite a reconstrução de um atrator representativo da dinâmica real do fenômeno. Os diagramas de fase foram reconstruídos a partir das respectivas séries temporais em duas dimensões com 8760 pontos e dimensão de imersão (n) otimizada igual 3. Em todos os casos fica claro que as linhas de fluxos definidas para as concentrações anuais de $\mathrm{PM}_{10}$ formam atratores estranhos, sugerindo assim uma dinâmica caótica. 

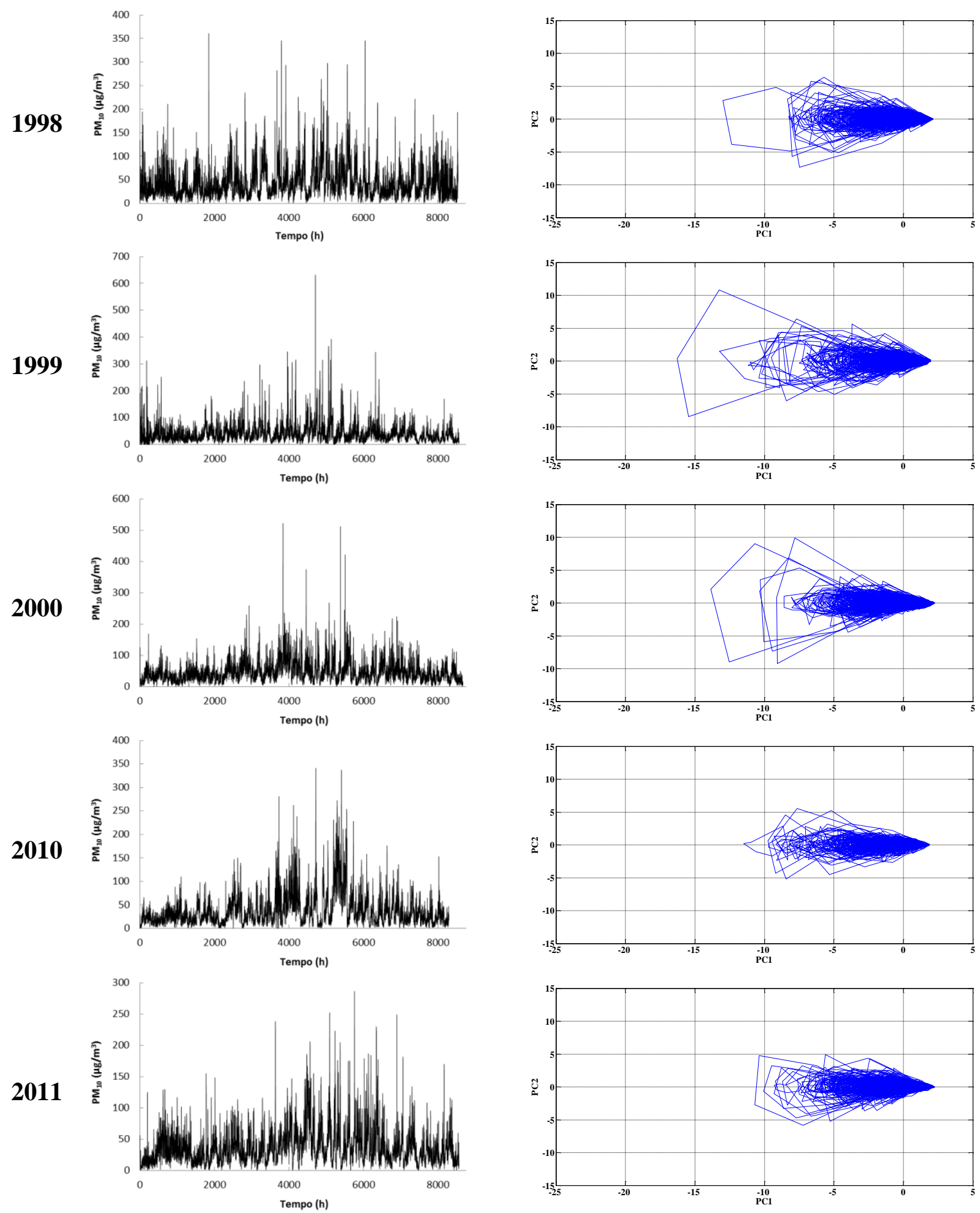
2012
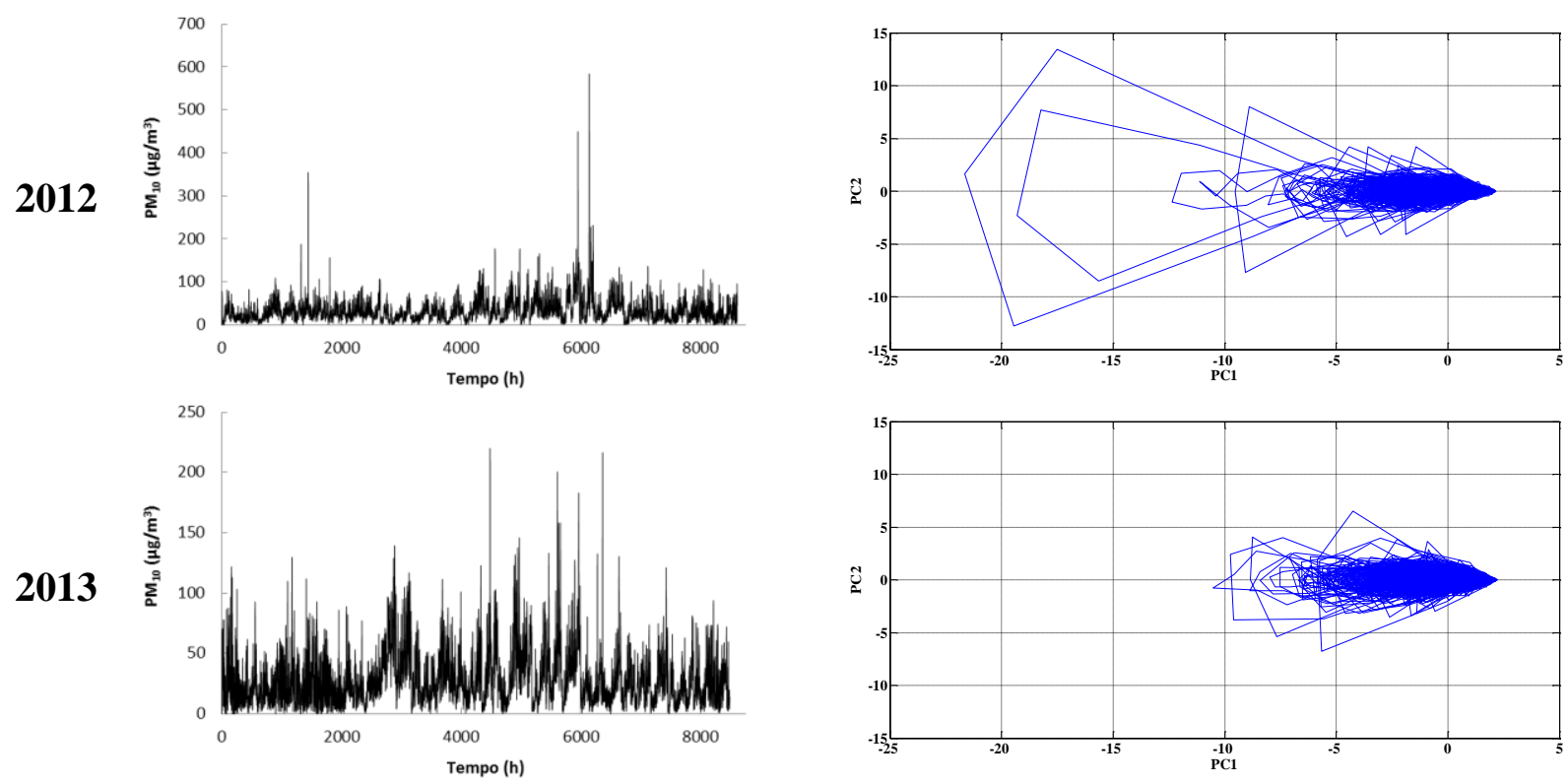

Figura 1 - Séries temporais e atratores da concentração de $\mathrm{PM}_{10}$.

Para dirimir dúvidas sobre a dinâmica dos dados analisados, apresenta-se a Tabela 1, na qual constam os valores da entropia de Kolmogorov para os anos estudados. Todas as séries temporais apresentaram caos, dado que para sistemas em que $\infty\langle K\rangle 0$ verifica-se a presença de dinâmica caótica. Os valores de entropia de Kolmogorov não revelaram nenhum perfil característico que permita associar os dados de entropia a um ano específico, pois os dados de concentração de $\mathrm{PM}_{10}$ são substancialmente afetados por fatores como condições meteorológicas (frequência e volume de precipitações, velocidade do ar etc.), implementação de políticas públicas e alterações imprevisíveis no local das medições. Todos estes fatores fazem com que as medições sejam mais ou menos aleatórias de ano para o outro.

Tabela 1- Entropia de Kolmogorov.

\begin{tabular}{cccccccc}
\hline Ano & 1998 & 1999 & 2000 & 2010 & 2011 & 2012 & 2013 \\
\hline $\begin{array}{c}\boldsymbol{K}_{M L} \\
\text { (bits/s) }\end{array}$ & 0,1944 & 0,2283 & 0,2211 & 0,1185 & 0,1909 & 0,2015 & 0,2179 \\
\hline
\end{tabular}

\subsection{Dimensão de Correlação}

Como a estrutura de muitos atratores estranhos é heterogênea e algumas de suas regiões são mais visitadas que outras, torna-se necessário o cálculo de dimensões que considerem as não homogeneidades presentes no espaço de fases e, neste sentido, a dimensão de correlação é uma ferramenta que determina o número de graus de liberdade do sistema avaliado. Pelo método de Grassberger e Procaccia (1983) é imperativo o conhecimento do tempo de atraso $(\theta)$. Na prática muitos autores utilizam a frequência de amostragem como o tempo de atraso (Glaser et al., 2011; Yu et al., 2013), sendo assim, no presente trabalho, utilizou-se $\theta=1$ hora. 
Os valores de $D_{2}$ variam com aumento da dimensão de imersão, sendo indispensável a avaliação do valor de convergência deste invariante. Para exemplificar, a Figura 2 apresenta a relação da integral de correlação $(C(\omega))$ com o raio $(\omega)$ e a convergência de $D_{2}$ com o aumento de $n$ para o ano de 1999. Neste caso, a convergência é atingida com $n=12$ e $D_{2}=4,40$. O número inteiro mais próximo determina a quantidade de variáveis que regem o sistema, sendo assim para os dados da Figura 2, observam-se quatro variáveis. Seguindo o mesmo procedimento para os anos de 1998, 2000, 2010 - 2013 se obtêm a Tabela 2.
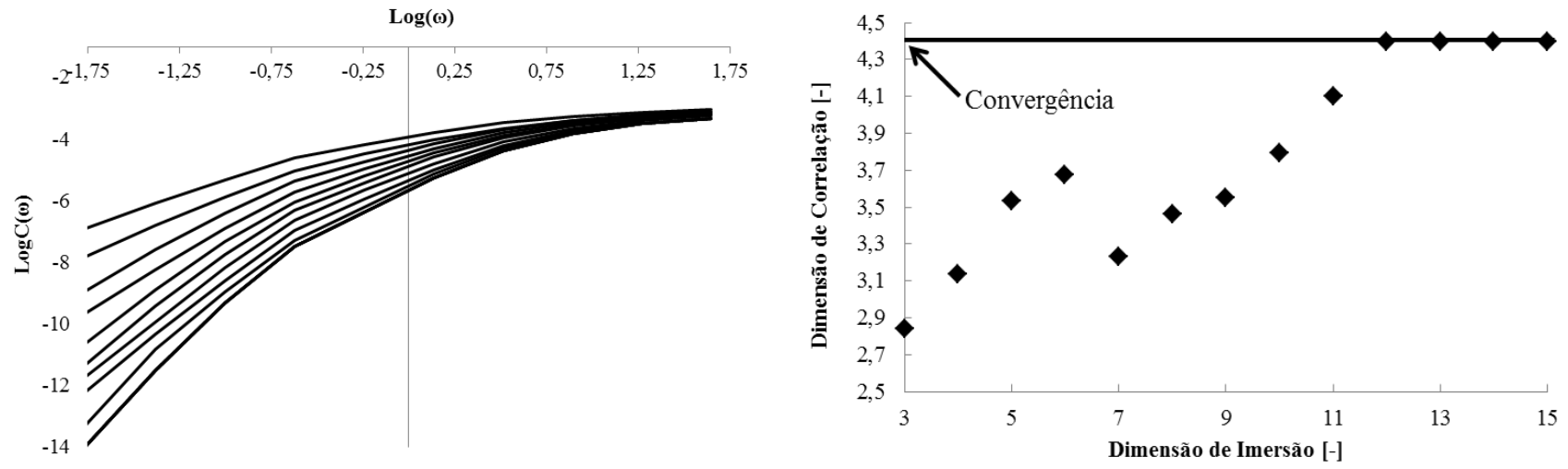

Figura 2 - Convergência da dimensão de correlação para concentração de PM 10 no ano de 1999.

Tabela 2 - Dimensão de correlação e graus de liberdade das séries temporais estudadas.

\begin{tabular}{cccc}
\hline Ano & $\boldsymbol{n}$ de convergência & $\mathbf{D}_{\mathbf{2}}[-]$ & Variáveis \\
\hline 1998 & 14 & 3,15 & 4 \\
1999 & 12 & 4,10 & 4 \\
2000 & 13 & 3,34 & 4 \\
2010 & 18 & 4,17 & 4 \\
2011 & 16 & 3,57 & 4 \\
2012 & 14 & 3,90 & 4 \\
2013 & 12 & 4,04 & 4 \\
\hline
\end{tabular}

Por inspeção da Tabela 2 verifica-se que, para as séries temporais relativas aos intervalos de 1998 à 2000 e 2010 à 2012, quatro variáveis regeram a determinação da dinâmica dos sistemas, porém observa-se uma diferença substancial nos valores de $\mathrm{D}_{2}$ de um ano para outro. Esta diferença ocorre devido à multiplicidade de fatores que, anualmente, influenciam de diferentes formas e intensidade estas observações. Independentemente disso, a dinâmica caótica é facilmente constatada pelos altos valores de dimensão de correlação. Considerando uma evolução temporal qualquer, baixas dimensões de correlação indicam a presença de atratores tipo pontos fixos no diagrama de fases. Transpondo tais pontos, ocorre a presença de ciclo limite e o subsequente aumento de $D_{2}$, indicando a presença de instabilidades que causam duplicação de período, culminando, desta feita, em dinâmica caótica para as dimensões maiores do que 2 e 3. Sendo assim, para todos os anos avaliados, constatou-se a presença do caos determinístico nas séries temporais de concentração de $\mathrm{PM}_{10}$. 


\section{9 a 22 de outubro de 2014 \\ Florianópolis/SC}

Fenomenologicamente as variações nas concentrações de $\mathrm{PM}_{10}$ estão relacionadas a fatores naturais e antropogênicos que variam constantemente tanto em frequência quanto em magnitude. Todos estes fatores impactam diretamente na interação do poluente com o ambiente e, portanto, sua medição, acarretando em diferentes dinâmicas temporais, mais ou menos complexas, incrementando dificuldade na identificação destas forças motrizes e de suas contribuições presentes na série temporal (Yu et al., 2013).

\section{CONCLUSÕES}

Com base nos valores de entropia de Kolmogorov e no de dimensão de correlação abstraídos dos atratores, nitidamente estranhos, verificou-se que as séries temporais de variação de concentração de material particulado inalável, medidas na estação de monitoramento do Ibirapuera, em São Paulo, nos anos de 1998 - 2000 e 2010 - 2013 apresentam clara dinâmica caótica, sendo assim possível estudá-las por meio da teoria caótica determinística. Com isso o caos determinístico pode ser aplicado para auxiliar no entendimento do fenômeno da poluição por meio da identificação das alterações dos padrões cíclicos ao longo do tempo, como também ser uma ferramenta para auxiliar na tomada de decisão na gestão da poluição atmosférica e no desenvolvimento de indicadores de poluição.

\section{REFERÊNCIAS}

BRIONGOS, J.V; SOLER, J.G. Using free bed surface fluctuations in a 3-D fluidized bed for dynamic characterization. AIChE Journal, vol 50, pp. 3060-3067, 2004.

BROOMHEAD, D. S.; KING, G. P. Extracting Qualitative Dynamics from Experimental Data. Physica 20D, pp. 217 - 236, 1986.

CASTILHO, G. J. Análise de Caos em Leito Fluidizado Circulante. Tese (Doutorado em Engenharia Química), Faculdade de Engenharia Química, Universidade Estadual de Campinas, Campinas-SP, 2011.

GLASER, S. M.; YE, H., MAUNDER, M.; MACCALL, A.; FOGARTY, M.; SUGIHARA, G., Detecting and forecasting complex nonlinear dynamics in spatially structured catch-per-unit-effort time series for North Pacific albacore (Thunnus alalunga). Can. J. Fish. Aquat. Sci. 68, 400e412, 2011.

GRASSBERGER, P.; PROCACCIA, I. Measuring the Strangeness of Strange Attractors. In Physica D, v. 9, pp. 189-208, 1983

GUO, H., 2011. Source apportionment of volatile organic compounds in Hong Kong homes. Build Environ. 46, 2280e2286.

HELlEBUST, S.; ALLANIC, A.; O’CONNOR, I. P.; WENGER, J. C.; SODEAU, J. R., 2010. The use of real-time monitoring data to evaluate major sources of airborne particulate matter. Atmos. Environ. 44, 1116e1125. 
LORENZ, E. N. Deterministic nonperiodic flow. J. Atmos. Sci., v. 20, pp. 130-141, 1963.

MCCAULEY, J. L. Introduction to multifractal in dynamical system theory and fully developed turbulence. Phys. Rev. 189, 225, 1990.

MONTEIRO, L. H. A. Sistemas Dinâmicos. $2^{\text {a }}$ Ed. São Paulo: Livraria da Física, 2011.

MOURA, H. L. Análise da influência do ruído na obtenção de atratores e parâmetros determinísticos da teoria do caos aplicada em uma seção de riser de um leito fluidizado circulante.Faculdade de Engenharia Química, Universidade Estadual de Campinas, Campinas-SP, 2013.

POPE, C. A. I.; BURNeTT, R. T.; THUN, M. J.; CALlE, E. E.; KREWSKI, D.; ITO, K.; THURSTON, G. D. 2002. Lung cancer, cardiopulmonary mortality, and long-term exposure to fine particulate air pollution. J. Am. Med. Assoc. 287, 1132e1141.

RUELLE, D. Chaotic evolution and strange attractors. Cambridge University Press: Cambridge, 1989.

RUELLE, D.; TAKENS, F. On the nature of turbulence, Commum. Math. Physics, v. 30, pp. 167192, 1971.

SAVI, M. A. Dinâmica Não Linear e Caos. Universidade Federal do Rio de Janeiro - COPPE, Engenharia Mecânica: [s.n.], 2004.

SCHOUTEN, J. C.; TAKENS, F.; VAN DEN BLEEK, C. M. Maximum-Likelihood-Estimation of the Entropy of an Attractor. In Physical Review E, v. 49, pp. 126-129, 1994.

TAKENS, F. Detecting strange attractors in turbulence, In: Dynamical systems and turbulence, Lecture Notes in Mathematics, Eds. D.A. Rand e L-S. Young, Springer Verlag, v. 898, pp.366-381, 1981.

TSONIS, A. A. Chaos: From Theory to Applications. New York: Plenum Press, 274 p. 1992.

VAN DEN BLEEK, C. M.; SCHOUTEN, J. C. Deterministic chaos: a new tool in fluidized bed design and operation. Chemical Engineering Journal, v. 53, pp. 75-87, 1993.

VANDER STAPPEN, MARIA, M. L. Chaotic hydrodynamics of fluidized beds, Delft: University of Technology, Delft - NL, 236 pp. PhD. Thesis, 1996.

YU, H-L.; LIN, Y-C.; SIVAKUMAR, B.; KUO, Y-M. A study of the temporal dynamics of ambient particulate matter using stochastic and chaotic techniques. Atmospheric Environment 69, 37-45, 2013. 